NoDEA

Nonlinear differ. equ. appl. 14 (2007) 233-257

1021-9722/07/040233-25

DOI $10.1007 / \mathrm{s} 00030-007-5012-5$ (c) Birkhäuser Verlag, Basel, 2007

Nonlinear Differential Equations

and Applications NoDEA

\title{
Hamilton-Jacobi methods for Vakonomic Mechanics
}

\author{
Diogo Aguiar Gomes \\ Instituto Superior Técnico Departamento de Matemática \\ Av. Rovisco Pais 1049-001 Lisboa, Portugal \\ e-mail: dgomes@math.ist.utl.pt
}

\begin{abstract}
We extend the theory of Aubry-Mather measures to Hamiltonian systems that arise in vakonomic mechanics and sub-Riemannian geometry. We use these measures to study the asymptotic behavior of (vakonomic) action-minimizing curves, and prove a bootstrapping result to study the partial regularity of solutions of convex, but not strictly convex, HamiltonJacobi equations.
\end{abstract}

2000 Mathematics Subject Classification: 37J50, 37J60, 49L25

Key words: Mother Theory, Hamilton-Jacobi Equations, Vakonomic Mechanics

\section{Introduction}

In classical (unconstrained) mechanics one looks for trajectories $x(t)$ that are minimizers (or critical points) of the action

$$
\int_{t_{0}}^{t_{1}} L(x, \dot{x}) d t
$$

in which $L$, the Lagrangian of the mechanical system, is the difference $L=T-V$ between the kinetic energy, $T(x, \dot{x})$, and potential energy $V(x)$. These trajectories are characterized by the Euler Lagrange equations

$$
\frac{d}{d t} \frac{\partial L}{\partial \dot{x}}-\frac{\partial L}{\partial x}=0 .
$$

Constrained (non-holonomic) systems appear naturally in mechanical problems. Non-holonomic systems satisfy the d'Alembert-Lagrange principle, and are described by equations of the form:

$$
\frac{d}{d t} \frac{\partial L}{\partial \dot{x}}-\frac{\partial L}{\partial x}=R(x, \dot{x}),
$$


in which $R$ represents the reaction forces that impose the constraint. Unfortunately, unless the constraint is integrable (holonomic case) these equations cannot be derived from a variational principle. Vakonomic mechanics is an alternative formulation to the non-holonomic mechanics [AKN97] based on a variational principle. In these systems, for each point $x$ in the configuration space there is a distribution $\mathcal{D}_{x}$ of velocities which is a finite dimensional subspace of $\mathbb{R}^{n}$. Then one looks for trajectories that are minimizers (or critical points) of (1) with the constraint that

$$
\dot{x}(t) \in \mathcal{D}_{x(t)},
$$

for all $t$. We should point out that these two approaches are not equivalent. As a model for mechanical systems, vakonomic systems correspond to realizing constraints using large anisotropic inertia, whereas d'Alembert-Lagrange principle corresponds to the large anisotropic friction limit [AKN97]. Furthermore, vakonomic mechanics has important connections with sub-Riemannian geometry [Str86], [Str89], [LS95], and is also an important model for control theory [FS93], [BCD97].

The integrability theory for classical unconstrained systems is well known, as well its connections with viscosity solutions of Hamilton-Jacobi equations [JKM99], [Fat97a], [Fat97b], [Fat98a], [Fat98b], [FS04], [FS05], [EG99a], [EG99b]. This paper is a first approach to study this problem for vakonomic systems in the framework of Aubry-Mather theory and viscosity solutions of Hamilton-Jacobi equations. We extend techniques from previous works (see references below) to use viscosity solution methods and construct generalizations of Aubry-Mather measures. As applications, we discuss the asymptotic behavior of vakonomic trajectories and the regularity theory for Hamilton-Jacobi (see also [EJ89], [EG99a], [Gom00]).

Let $\mathbb{T}^{n}=\mathbb{R}^{n} / \mathbb{Z}^{n}$ be the $n$-dimensional torus. A smooth distribution $\mathcal{D}$ is a subset of the tangent space of $T \mathbb{T}^{n}$ that at each point $x \in \mathbb{T}^{n}$ is a constant rank linear subspace $\mathcal{D}_{x}$ of $T_{x} \mathbb{T}^{n}$, furthermore $\mathcal{D}_{x}$ depends smoothly on $x$. We assume that the rank of $\mathcal{D}_{x}$ is constant $m \leq n$ and that there exist vector fields $f^{1}, \cdots, f^{m}$ such that

$$
\mathcal{D}_{x}=\operatorname{span}\left\{f^{1}, \cdots, f^{m}\right\} .
$$

Additionally, without loss of generality, we suppose that $f^{1}, \cdots, f^{m}$ are orthonormal with respect to some metric in $T \mathbb{T}^{n}$. In local coordinates the metric is given by its components $g^{i j}$ and we have

$$
g^{i j} f_{i}^{k} f_{j}^{l}=\delta^{k l}
$$

We assume that the metric $g^{i j}$ is definite positive in $T \mathbb{T}^{n}$, that is, for some $\theta>0$

$$
g^{i j} \chi_{i} \chi_{j} \geq \theta|\chi|^{2}, \quad \forall \chi \in \mathbb{R}^{n}
$$


A Lipschitz curve $x(\cdot):[0, T] \rightarrow \mathbb{T}^{n}$ is compatible with $\mathcal{D}$, if for almost every $t \in[0, T]$,

$$
\dot{x}(t) \in \mathcal{D}_{x(t)},
$$

that is, there exists, for almost every $t \in[0, T]$, a vector $v \in \mathbb{R}^{m}$ such that

$$
\dot{x}_{k}(t)=f_{k}^{i}(x) v_{i} .
$$

Given a Lagrangian

$$
L: T \mathbb{T}^{n} \rightarrow \mathbb{R}
$$

we would like to study the trajectories $x(\cdot):[a, b] \rightarrow \mathbb{T}^{n}$ that minimize the action

$$
\int_{a}^{b} L(x, \dot{x}) d s
$$

and are compatible with the distribution $\mathcal{D}$. Such trajectories are called vakonomic trajectories.

A particularly important class of Lagrangians $L$ has the form

$$
L(x, \dot{x})=\frac{1}{2} g^{i j}(x) \dot{x}_{i} \dot{x}_{j}-V(x),
$$

the first term is the kinetic energy and $V(x)$ is the potential energy. We assume through this paper that $L$ is given by the above expression, or is a related Lagrangian

$$
L_{P}(x, \dot{x})=L(x, \dot{x})+P \dot{x}
$$

for $P \in \mathbb{R}^{n}$

Since $V$ is bounded we may add any constant to $L$ without changing the minimization problem. Therefore we may assume without loss of generality that $L \geq 0$.

Vakonomic trajectories for such Lagrangians were characterized completely in [KO01]. Our objective in this paper is somewhat different - we consider generalized vakonomic trajectories (measures supported on $\mathcal{D}$ ) and develop an analog of Aubry-Mather theory. Our methods will be a combination of dynamical systems ideas with viscosity solutions of Hamilton-Jacobi equations.

Let the Hamiltonian $H$ be defined by

$$
H(p, x)=\sup _{v \in \mathbb{R}^{m}}\left[p^{i} f_{i}^{k} v_{k}-L\right]=\frac{1}{2}|p f|^{2}+V(x) .
$$

The Hamiltonian dynamics associated to $H$ is given by the Hamilton's equations:

$$
\dot{x}=-D_{p} H(p, x) \quad \dot{p}=D_{x} H(p, x) .
$$

A classical procedure to integrate these equations [AKN97] is to build a a smooth solution $u(x, P)$ of the Hamilton-Jacobi equation:

$$
H\left(P+D_{x} u, x\right)=\bar{H}(P)
$$


with $\bar{H}(P)$ being the unique value for which the equation admits a periodic solution $u$. Then this solution is a generating function that yields a change of coordinates $X(p, x)$ and $P(p, x)$ defined by the equations:

$$
p=P+D_{x} u \quad X=x+D_{P} u .
$$

This change of coordinates simplifies (2) into:

$$
\dot{P}=0 \quad \dot{X}=-D_{P} \bar{H}(P) .
$$

In other words, this means that for each $P$ there is an invariant torus in which the dynamics is simply a rotation. However, (3) does not admit, in general, smooth solutions and this procedure is not valid. Nevertheless, as we show in this paper, one can recover weak analogs of the classical integrability using viscosity solutions methods.

One of the most important cases is the one in which the distribution satisfies an Hörmander-type condition, that is, $f^{1}, \cdots, f^{m}$ together with finitely many brackets $\left[f^{i}, f^{j}\right],\left[\left[f^{i}, f^{j}\right], f^{k}\right], \ldots$ span $T_{x} \mathbb{T}^{n}$ at each point. If this condition is fulfilled, we say that $\mathcal{D}$ is bracket generating. Under this condition we will show that one can construct a viscosity solution to (3) that enjoys a limited regularity (Hölder continuity).

If $\mathcal{D}$ is bracket generating the vakonomic system is controllable, i.e., given two points $x, y \in \mathbb{T}^{n}$ and any time $T$ there exists a compatible trajectory $\gamma$ : $[0, T] \rightarrow \mathbb{T}^{n}$ with $\gamma(0)=x$ and $\gamma(T)=y$ [Cho39] (see also Carathéodory [Car09], and $[\mathrm{Bac} 86])$.

The outline of this paper is the following: in section 2 we prove the existence of viscosity solution of stationary Hamilton-Jacobi equations, and study the Hölder regularity of such solutions. In section 3 we introduce generalized Mather measures and use duality theory to connect the minimal measure problem with viscosity solutions of Hamilton-Jacobi equations. Section 4 is dedicated to the characterization of the support of the generalized Mather measure. Finally in section 5 we consider some applications, the asymptotics of vakonomic trajectories and the partial regularity for solutions of Hamilton-Jacobi equations.

\section{$2 \quad$ Ergodic and stationary problems}

In this section we study the existence and regularity properties of viscosity solutions that arise from control problems related to vakonomic mechanics. Since some of the readers may not be familiar with viscosity solutions, we first recall the definition of viscosity solution, however for more details and related material, one should consult, for instance, [FS93] or [BCD97].

Let $F$ be a continuous function. A function $u$ is a viscosity solution of the Hamilton-Jacobi equation

$$
F\left(D_{x} u, u, x\right)=0
$$


provided that for any smooth function $\phi$ such $u-\phi$ has a local maximum (resp. minimum) at a point $x_{0}$ then $F\left(D_{x} \phi\left(x_{0}\right), u\left(x_{0}\right), x_{0}\right) \leq 0$ (resp. $\geq 0$ ).

The discounted cost infinite horizon problem consists in finding the value function $u^{\alpha}$ defined by

$$
u^{\alpha}(x)=\inf _{x(0)=x} \int_{0}^{+\infty} e^{-\alpha t} L(x, \dot{x})+P \dot{x},
$$

in which the infimum is take over all trajectories that satisfy $x(t) \in \mathcal{D}_{x(t)}$, that is,

$$
\dot{x}_{k}=f_{k}^{i}(x) u_{i}
$$

for some bounded control $u(\cdot):[0,+\infty) \rightarrow \mathbb{R}^{m}$.

Proposition $1 u^{\alpha}$ is a periodic viscosity solution of

$$
\alpha u^{\alpha}+H\left(P+D_{x} u^{\alpha}, x\right)=0 .
$$

\section{Furthermore}

$$
-C \leq \alpha u^{\alpha} \leq C
$$

uniformly in $\alpha$.

Proof. $u^{\alpha}$ is a periodic viscosity solution, using standard results in viscosity solutions theory [FS93]. Since $L(x, \dot{x})+P \dot{x} \geq-C$ (because $L$ grows quadratically in $\dot{x}$ ) we have $\alpha u^{\alpha} \geq-C$. Choosing the constant trajectory $\dot{x}=0$, which is admissible, we obtain (5).

Before stating and proving the next result it is convenient to recall the properties of the sup and inf convolutions ([JLS88], and [FS93]). Given a bounded function $u$ define the sup convolution $u^{\epsilon}$ by

$$
u^{\epsilon}(x)=\sup _{y}\left[u(y)-\frac{|x-y|^{2}}{\epsilon^{2}}\right] .
$$

Similarly the inf convolution is given by

$$
u_{\epsilon}(x)=\inf _{y}\left[u(y)+\frac{|x-y|^{2}}{\epsilon^{2}}\right] .
$$

Proposition 2 (Properties of sup and inf convolutions)

1. $u^{\epsilon}$ and $u_{\epsilon}$ are, respectively, semiconvex and semiconcave.

2. $\left|D_{x} u^{\epsilon}\right|,\left|D_{x} u_{\epsilon}\right| \leq \frac{C}{\epsilon}$. 
3. If $u$ is a viscosity solution of $H\left(D_{x} u, x\right)=\bar{H}$ then $u^{\epsilon}$ and $u_{\epsilon}$ satisfies, respectively,

$$
H\left(D_{x} u^{\epsilon}, x\right) \leq \bar{H}+O(\epsilon), \quad H\left(D_{x} u_{\epsilon}, x\right) \geq \bar{H}+O(\epsilon)
$$

almost everywhere and in the viscosity sense.

Proof. The proof of these statements can be found in [FS93], pages 241-243.

Proposition 3 Assume $\mathcal{D}$ is bracketing generating, then $u^{\alpha}$ is Hölder continuous uniformly in $\alpha$.

Proof. The proof of this result when $f^{i}$ and $\left[f^{i}, f^{j}\right]$ span $T \mathbb{T}^{n}$ can be found in [EJ89]. Using similar techniques we sketch the proof in the general case. The proposition is a corollary of the following lemma:

Lemma 1 Suppose that $\mathcal{D}$ is bracketing generating and $\left|f_{k} D_{x} u\right| \leq C$ in the viscosity sense. Then $u$ is Hölder continuous of exponent $\beta=\frac{1}{k+1}$ in which $k$ is the number of brackets required to generate $T \mathbb{T}^{n}$.

Proof. First step: give the viscosity solution define $u^{\epsilon}$ as in (6). Then, as in [EJ89], we have

$$
\left|D_{x} u^{\epsilon}\right| \leq \frac{C}{\epsilon} \quad\left|f_{k} D_{x} u^{\epsilon}\right| \leq C,
$$

with the constants not depending on $\epsilon$.

Define $X^{k}(t): \mathbb{T}^{n} \rightarrow \mathbb{T}^{n}$ to be the time- $t$ flow map corresponding to the differential equation

$$
\dot{x}=f_{k}(x) \quad x(0)=x .
$$

Note that

$$
\left|u^{\epsilon}(x)-u^{\epsilon}\left(X^{k}(t) x\right)\right| \leq C|t|,
$$

for all sufficiently small $t$, uniformly in $\epsilon$. Observe that

$$
X^{k}(t)(x)=x+f_{k}(x) t+O\left(t^{2}\right),
$$

as $t \rightarrow 0$. For each commutator $\left[f_{i_{1}}, f_{i_{2}}\right]$ define the vector field, for $t$ small,

$$
X^{i_{1}, i_{2}}(t)=X^{i_{1}}\left(t^{1 / 2}\right) X^{i_{2}}\left(t^{1 / 2}\right) X^{i_{1}}\left(-t^{1 / 2}\right) X^{i_{2}}\left(-t^{1 / 2}\right)
$$

note that

$$
X^{i_{1}, i_{2}}(t)(x)=x+\left[f_{i_{1}}, f_{i_{2}}\right](x) t+O\left(t^{3 / 2}\right) .
$$

By induction define (for $t<0$ we use the convention $t^{\alpha}=-|t|^{\alpha}$ )

$$
\begin{aligned}
X^{i_{1}, i_{2}, \cdots, i_{m}}(t)= & X^{i_{1}, i_{2}, \cdots, i_{m-1}}\left(t^{(m-1) / m}\right) X^{i_{m}}\left(t^{1 / m}\right) \\
& X^{i_{1}, i_{2}, \cdots, i_{m-1}}\left(-t^{(m-1) / m}\right) X^{i_{m}}\left(-t^{1 / m}\right) .
\end{aligned}
$$


We claim that

$$
X^{i_{1}, i_{2}, \cdots, i_{m}}(t)(x)=x+\left[\cdots\left[\left[f_{i_{1}}, f_{i_{2}}\right], \cdots\right], f_{i_{m}}\right] t+O\left(t^{(m+1) / m}\right) .
$$

For the proof of this claim see [BCD97] (Lemma 1.19, p. 236) or [HH70].

As in [EJ89], we have, for $t$ sufficiently small,

$$
\left|u^{\epsilon}\left(X^{i_{1}, i_{2}, \cdots, i_{m}}(t)(x)\right)-u^{\epsilon}(x)\right| \leq C t^{1 / m}
$$

Define maps $Y_{j}(t)=X^{i_{1}^{j}, \cdots, i_{m_{j}}^{j}}(t), j=1, \cdots, n$, in such a way that $\frac{d}{d t} Y_{j}$ spans $T \mathbb{T}^{n}$. This is possible because of the hypothesis on the vector fields. Note that

$$
Y(s)=Y_{1}\left(s_{1}\right) \cdots Y_{n}\left(s_{n}\right) \quad s \in \mathbb{R}^{n}
$$

defines, by the implicit function theorem, a (local) diffeomorphism from $\mathbb{R}^{n}$ to $\mathbb{T}^{n}$ for $s$ sufficiently small. Thus for $y$ sufficiently close to $x$ we have

$$
\left|u^{\epsilon}(y)-u^{\epsilon}(x)\right|=\left|u^{\epsilon}(Y(s) x)-u^{\epsilon}(x)\right| \leq C|y-x|^{1 /(k+1)}
$$

in which $k$ is the number of brackets necessary to span $T \mathbb{T}^{n}$.

As a corollary of this proof we also have: if $v:[0, T] \rightarrow \mathbb{R}^{m}$ is bounded and $x(t)$ is a trajectory of

$$
\dot{x}=f(x) v(t)
$$

then $|u(x(t))-u(x(0))| \leq C|t|$, that is, $u$ is Lipschitz along compatible trajectories.

Furthermore the proof also shows that for any $x$ there exists an open set of points $y$ such that there is a compatible trajectory connecting $x$ to $y$ in finite time (actually this is a particular case of Chow's Theorem [Cho39] mentioned at the introduction, see also [Bac86]).

Related results for minimal time problems can be found in [Liv80], [Ran82], [Gyu84], [Gyu87], and [Sor92].

Corollary 1 For almost every $x$ there exists the directional derivative along a curve whose tangent is $f(x) v$, for any $v \in \mathbb{R}^{m}$

Proof. Observe that

$$
\left|u\left(X^{k}(t)(x)\right)-u(x)\right| \leq C|t|
$$

thus $u$ is Lipschitz along this curve and so has a directional derivative (along the curve) for almost every $t$. Then applying Fubinni's theorem yields that for a.e. $x$ such derivative exists.

Theorem 1 There exists a viscosity solution $u$ of the equation

$$
H\left(P+D_{x} u, x\right)=\bar{H}(P) .
$$

This solution is Hölder continuous. 
Proof. Since $\alpha u^{\alpha}$ is bounded and $u^{\alpha}$ is periodic and uniformly Hölder continuous we get that through some subsequence $\alpha u^{\alpha} \rightarrow-\bar{H}$, for some number $\bar{H}$. Also $u^{\alpha}-\min u^{\alpha}$ is periodic, uniformly bounded and Hölder, through some subsequence it converges to a periodic Hölder continuous function $u$. Standard stability results for viscosity solutions imply that $u$ is a viscosity solution of (8).

Proposition 4 For almost every $x$ we have

$$
H\left(P+D_{x} u, x\right)=\bar{H}(P) .
$$

Remark. Note that the derivative may not exist for almost every $x$ but the Hamiltonian only depends on the directional derivative.

Proof. See [BCD97], Theorem 2.40, p. 128.

Finally we discuss the uniqueness of $\bar{H}(P)$.

Theorem $2 \bar{H}(P)$ is unique.

Proof. Suppose $u_{1}$ and $u_{2}$ are viscosity solutions of

$$
H\left(P+D_{x} u_{i}, x\right)=\bar{H}_{i}(P) \quad(i=1,2) .
$$

Assume $H_{1}(P)>\bar{H}_{2}(P)$ and by adding constants, if necessary, we may also suppose that $0 \leq u_{2}<u_{1}$. For $\epsilon$ small enough

$$
H\left(P+D_{x} u_{1}, x\right)+\epsilon u_{1}>H\left(P+D_{x} u_{2}, x\right)+\epsilon u_{2}
$$

The comparison principle (see [BCD97], Theorem 2.12, p. 107) implies $u_{1} \leq u_{2}$, which is a contradiction.

\section{$3 \quad$ Duality and generalized Mather measures}

In this section, using the duality techniques from [Gom02] we develop a generalization of Mather measures to vakonomic systems.

We say that a positive probability measure $\mu$ on $\mathcal{D}$ is a vakonomic measure if for any $\phi \in C^{1}\left(\mathbb{T}^{n}\right)$ we have

$$
\int_{\mathcal{D}} D_{x} \phi f(x) v d \mu(x, v)=0 .
$$

The motivation for this definition is as follows: suppose $v: \mathbb{T}^{n} \rightarrow \mathbb{R}^{m}$ is a given function. Let $x(\cdot)$ be a solution of

$$
\dot{x}=f(x) v(x) .
$$


Define a probability measure $\mu$ on $\mathcal{D}$ by

$$
\lim _{T \rightarrow \infty} \frac{1}{T} \int_{0}^{T} \psi(x(t), \dot{x}(t)) d t \rightarrow \int_{\mathcal{D}} \psi(x, f v) d \mu,
$$

through some subsequence, for all bounded continuous functions $\psi: T \mathbb{T}^{n} \rightarrow \mathbb{R}$. Furthermore note that

$$
\frac{1}{T} \int_{0}^{T} D_{x} \phi(x) \dot{x}=O\left(\frac{1}{T}\right)
$$

for any $\phi \in C^{1}\left(\mathbb{T}^{n}\right)$. Thus $\int_{\mathbb{T}^{n} \times \mathbb{R}^{m}} D_{x} \phi f(x) v d \mu(x, v)$. Thus vakonomic measures should be though of as generalized compatible curves.

Let $\mathcal{M}$ be the set of signed Radon measures such that

$$
\int \gamma d|\mu| \leq \infty
$$

in which $\gamma: \mathbb{R}^{m} \rightarrow[1,+\infty)$ is a weight function that satisfies

$$
\frac{L(x, f v)}{\gamma(v)} \rightarrow+\infty \quad \frac{|v|}{\gamma(v)} \rightarrow 0,
$$

as $|v| \rightarrow+\infty$. Note that $\mathcal{M}$ is the dual of $C_{\gamma}^{0}(\mathcal{D})$, that is the set of continuous functions $\psi \in \mathcal{D}$ that satisfy

$$
\lim _{|v| \rightarrow \infty} \frac{\psi(x, v)}{\gamma(|v|)} \rightarrow 0
$$

The advantage of using the weight function $\gamma$ is that then $v D_{x} \phi(x) \in C_{\gamma}^{0}$, and so the constraint (9) is continuous, that is, if $\mu_{n} \rightarrow \mu$ and $\int_{\mathcal{D}} v D_{x} \phi(x) d \mu_{n}=0$ then $\int_{\mathcal{D}} v D_{x} \phi(x) d \mu=0$.

Consider also the following subsets of $\mathcal{M}$ :

$$
\mathcal{M}_{0}=\{\mu \in \mathcal{M}: \mu \text { is a vakonomic measure }\}
$$

and

$$
\mathcal{M}_{1}=\left\{\mu \in \mathcal{M}: \mu \geq 0, \int d \mu=1\right\} .
$$

The minimization problem that we wish to consider is

$$
\inf _{\mathcal{M}_{0} \cap \mathcal{M}_{1}} \int_{\mathcal{D}} L d \mu .
$$

If $\mathcal{D}=T \mathbb{T}^{n}$ then this is Mather's minimal measure problem, as formulated by Mañe [Mn96]. The next lemma is taken from [Mn96]: 
Lemma 2 (Mañe) For any constant $C$ the set of measures $\mu \in \mathcal{M}_{0} \cap \mathcal{M}_{1}$ that satisfy

$$
\int L d \mu \leq C
$$

is compact with respect to the weak-* topology in $\left(C_{\gamma}^{0}\right)^{\prime}$.

Using this lemma we prove the existence of a minimal measure:

Theorem 3 There exists a minimal measure $\mu \in \mathcal{M}_{0} \cap \mathcal{M}_{1}$ such that

$$
\inf _{\mathcal{M}_{0} \cap \mathcal{M}_{1}} \int_{\mathcal{D}} L d \mu=\int L d \mu .
$$

Furthermore this measure is supported on a graph $(x, v(x))$.

Proof. Suppose $\mu_{n}$ is a minimizing sequence in $\mathcal{M}_{0} \cap \mathcal{M}_{1}$ such that

$$
\int_{\mathcal{D}} L d \mu_{n} \rightarrow \inf _{\mathcal{M}_{0} \cap \mathcal{M}_{1}} \int_{\mathcal{D}} L d \mu .
$$

Since the infimum is finite $\int L d \mu_{n} \leq C$ for some constant $C$ independent of $n$. The previous lemma implies $\mu_{n} \stackrel{*}{\rightarrow} \mu$ for some measure $\mu$, through some subsequence. Then for any fixed $k$

$$
\int_{\mathcal{D}} \min \{L, k\} d \mu_{n} \rightarrow \int_{\mathcal{D}} \min \{L, k\} d \mu .
$$

Thus

$$
\int_{\mathcal{D}} \min \{L, k\} d \mu \leq \inf _{\mathcal{M}_{0} \cap \mathcal{M}_{1}} \int_{\mathcal{D}} L d \mu
$$

The monotone convergence theorem implies

$$
\int_{\mathcal{D}} L d \mu \leq \inf _{\mathcal{M}_{0} \cap \mathcal{M}_{1}} \int_{\mathcal{D}} L d \mu .
$$

By contradiction assume that $\mu$ is not supported on a graph $(x, v(x))$. Construct a measure $\tilde{\mu}$ supported in the graph $(x, v(x))$ with $v(x)$ being the unique function (defined $\mu$ almost everywhere) for which

$$
\int_{\mathcal{D}} v(x) \phi(x) d \mu=\int_{\mathcal{D}} v \phi(x) d \mu,
$$

and

$$
\int_{\mathcal{D}} \psi(x, v) d \tilde{\mu}=\int_{\mathcal{D}} \psi(x, v(x)) d \tilde{\mu} .
$$

Then $\tilde{\mu}$ is a probability measure that satisfies (9). Since $L$ is strictly convex in $v$ the average action of $\tilde{\mu}$ is smaller than the one of $\mu$, which is a contradiction. 
This minimization problem is a convex linear programming problem over a space of Radon measures. Related control problems have been studied by duality methods [VL78a], [VL78b], [LV80], [FV89], [FV88] and [Fle89], in which FenchelRockafellar duality Theorem [Roc66] is used to analyze optimal control problems. Using Legendre-Fenchel duality theory we obtain:

Theorem 4 We have

$$
-\inf _{\mu \in \mathcal{M}} \int_{\mathcal{D}} L d \mu=\inf _{\phi} \sup _{x} H\left(D_{x} \phi, x\right) .
$$

Remark. In the case in which $\mathcal{D}=T \mathbb{T}^{n}$ (or more generally the tangent space of a Riemannian manifold) this identity is proved in [CIPP98].

Before proving this theorem we need some preliminary results and definitions.

Suppose that $E$ is a Banach space with dual $E^{\prime}$ with the dual pairing between $E$ and $E^{\prime}$ denoted by $(\cdot, \cdot)$. Let $h_{1}: E \rightarrow(-\infty,+\infty]$ be a convex, lower semicontinuous function. The Legendre-Fenchel transform $h_{1}^{*}: E^{\prime} \rightarrow[-\infty,+\infty]$ of $h_{1}$ is defined by

$$
h_{1}^{*}(y)=\sup _{x \in E}\left(-(x, y)-h_{1}(x)\right),
$$

for $y \in E^{\prime}$. For concave, upper semicontinuous functions $h_{2}: E \rightarrow(-\infty,+\infty]$ the Legendre-Fenchel transform $h_{2}^{*}$ is defined similarly by

$$
h_{2}^{*}(y)=\inf _{x \in E}\left(-(x, y)-h_{2}(x)\right) .
$$

A classical duality result is the Legendre-Fenchel-Rockafellar duality theorem that we quote next:

Theorem 5 (Rockafellar [Roc66]) Let E be a locally convex Hausdorff topological vector space over $\mathbb{R}$ with dual $E^{*}$. Suppose $h_{1}: E \rightarrow(-\infty,+\infty]$ is convex and lower semicontinuous, $h_{2}: E \rightarrow[-\infty,+\infty)$ is concave and upper semicontinuous. Then

$$
\sup _{x} h_{2}(x)-h_{1}(x)=\inf _{y} h_{1}^{*}(y)-h_{2}^{*}(y),
$$

provided that either $h_{1}$ or $h_{2}$ is continuous at some point where both functions are finite.

To use this theorem to prove identity (10) we define two functions $h_{1}$ and $h_{2}$ on $C_{\gamma}^{0}(\mathcal{D}) \sim C_{\gamma}^{0}\left(\mathbb{T}^{n} \times \mathbb{R}^{m}\right)$ and compute the dual problem of

$$
\sup _{\phi \in C_{\gamma}^{0}(\mathcal{D})} h_{2}(\phi)-h_{1}(\phi) .
$$

The first function $h_{1}$ is defined by

$$
h_{1}(\phi)=\sup _{(x, v) \in \mathcal{D}}[-\phi(x, v)-L(x, v)] .
$$


Let

$$
\mathcal{C}=\operatorname{cl}\left\{\phi: \phi(x, v)=D_{x} \varphi(x) f(x) v, \varphi \in C^{1}\left(\mathbb{T}^{n}\right)\right\}
$$

and set

$$
h_{2}(\phi)= \begin{cases}0 & \text { if } \phi \in \mathcal{C} \\ -\infty & \text { otherwise }\end{cases}
$$

Proposition 5 We have

$$
h_{1}^{*}(\mu)= \begin{cases}\int L d \mu & \text { if } \mu \in \mathcal{M}_{1} \\ +\infty & \text { otherwise }\end{cases}
$$

and

$$
h_{2}^{*}(\mu)= \begin{cases}0 & \text { if } \mu \in \mathcal{M}_{0} \\ -\infty & \text { otherwise. }\end{cases}
$$

Remark. This proof is is very similar to the one in [Gom02], the only main difference being the functions $h_{1}$ and $h_{2}$. This shows how general these duality methods are to handle generalizations of the Aubry-Mather theory and relate them to Hamilton-Jacobi equations.

Proof. The Legendre-Fenchel transform $h_{1}^{*}$ of $h_{1}$ is

$$
h_{1}^{*}(\mu)=\sup _{\phi \in C_{\gamma}^{0}(\mathcal{D})}\left(-\int \phi d \mu-h_{1}(\phi)\right) .
$$

We claim that for all non-positive measures $\mu, h_{1}^{*}(\mu)=\infty$.

Lemma 3 If $\mu \nsupseteq 0$ then $h_{1}^{*}(\mu)=+\infty$.

Proof. If $\mu \nsupseteq 0$ there is a sequence of non-negative functions $\phi_{n} \in C_{\gamma}^{0}(\mathcal{D})$ such that

$$
\int-\phi_{n} d \mu \rightarrow+\infty
$$

Thus, since $L \geq 0$,

$$
\sup _{\mathcal{D}}-\phi_{n}-L \leq 0 .
$$

If $\mu \nsupseteq 0$, we have $h_{1}^{*}(\mu)=+\infty$.

Lemma 4 If $\mu \geq 0$ then

$$
h_{1}^{*}(\mu) \geq \int L d \mu+\sup _{\psi \in C_{\gamma}^{0}(\mathcal{D})}\left(\int \psi d \mu-\sup \psi\right) .
$$


Proof. Let $L_{n}$ be a sequence of functions in $C_{\gamma}^{0}(\mathcal{D})$ increasing pointwise to $L$. Any function $\phi$ in $C_{\gamma}^{0}(\mathcal{D})$ can be written as $\phi=-L_{n}-\psi$, for some $\psi$ also in $C_{\gamma}^{0}(\mathcal{D})$. Therefore

$$
\begin{aligned}
\sup _{\phi \in C_{\gamma}^{0}(\mathcal{D})} & \left(-\int \phi d \mu-h_{1}(\phi)\right) \\
= & \sup _{\psi \in C_{\gamma}^{0}(\mathcal{D})}\left(\int L_{n} d \mu+\int \psi d \mu-\sup \left(L_{n}+\psi-L\right)\right) .
\end{aligned}
$$

Note that $L_{n}-L \leq 0$ implies

$$
\sup _{\mathcal{D}} L_{n}-L \leq 0
$$

thus

$$
\sup _{\mathcal{D}}\left(L_{n}+\psi-L\right) \leq \sup _{\mathcal{D}} \psi
$$

Therefore

$$
\sup _{\phi \in C_{\gamma}^{0}(\mathcal{D})}\left(-\int \phi d \mu-h_{1}(\phi)\right) \geq \sup _{\psi \in C_{\gamma}^{0}(\mathcal{D})}\left(\int L_{n} d \mu+\int \psi d \mu-\sup (\psi)\right) .
$$

By the monotone convergence theorem $\int L_{n} d \mu \rightarrow \int L d \mu$, and so

$$
\sup _{\phi \in C_{\gamma}^{0}(\mathcal{D})}\left(-\int \phi d \mu-h_{1}(\phi)\right) \geq \int L d \mu+\sup _{\psi \in C_{\gamma}^{0}(\mathcal{D})}\left(\int \psi d \mu-\sup (\psi)\right),
$$

as required.

If $\int L d \mu=+\infty$ then $h_{1}^{*}(\mu)=+\infty$. Also if $\int d \mu \neq 1$ then

$$
\sup _{\psi \in C_{\gamma}^{0}(\mathcal{D})}\left(\int \psi d \mu-\sup \psi\right) \geq \sup _{\alpha \in \mathbb{R}} \alpha\left(\int d \mu-1\right)=+\infty,
$$

by taking $\psi \equiv \alpha$, constant. So, $h_{1}^{*}(\mu)=+\infty$, and therefore a finite value of $h_{1}^{*}$ is only possible if $\int d \mu=1$.

If $\int d \mu=1$ we have, from the previous lemma,

$$
h_{1}^{*}(\mu) \geq \int L d \mu,
$$

by taking $\psi \equiv 0$.

Also, for any function $\phi$

$$
\int(-\phi-L) d \mu \leq \sup _{\mathcal{D}}(-\phi-L),
$$


if $\int d \mu=1$. Hence

$$
\sup _{\phi \in C_{\gamma}^{0}(\mathcal{D})}\left(-\int \phi d \mu-h_{1}(\phi)\right) \leq \int L d \mu .
$$

Thus

$$
h_{1}^{*}(\mu)= \begin{cases}\int L d \mu & \text { if } \mu \in \mathcal{M}_{1} \\ +\infty & \text { otherwise. }\end{cases}
$$

Now we will compute $h_{2}^{*}$. First observe that if $\mu \notin \mathcal{M}_{0}$ then there exists $\hat{\phi} \in \mathcal{C}$ such that

$$
\int \hat{\phi} d \mu \neq 0
$$

and so

$$
\inf _{\phi \in \mathcal{C}}-\int \phi d \mu \leq \inf _{\alpha \in \mathbb{R}} \alpha \int \hat{\phi} d \mu=-\infty .
$$

If $\mu \in \mathcal{M}_{0}$ then $\int \phi d \mu=0$, for all $\phi \in \mathcal{C}$. Therefore

$$
h_{2}^{*}(\mu)=\inf _{\phi \in \mathcal{C}}-\int \phi d \mu= \begin{cases}0 & \text { if } \mu \in \mathcal{M}_{0} \\ -\infty & \text { otherwise. }\end{cases}
$$

Theorem 5 yields then

$$
\sup _{\phi \in C_{\gamma}^{0}(\mathcal{D})} h_{2}(\phi)-h_{1}(\phi)=\inf _{\mu \in \mathcal{M}} h_{1}^{*}(\mu)-h_{2}^{*}(\mu),
$$

provided we prove that $h_{1}$ is continuous on the set $h_{2}>-\infty$. This is the content of the next lemma.

Lemma $5 h_{1}$ is continuous.

Proof. Suppose $\phi_{n} \rightarrow \phi$ in $C_{\gamma}^{0}$. Then $\left\|\phi_{n}\right\|_{\gamma}$ and $\|\phi\|_{\gamma}$ are bounded uniformly by some constant $C$. The growth condition on $L$ implies that there exists $R>0$ such that

$$
\sup _{\mathcal{D}}-\hat{\phi}-L=\sup _{T^{n} \times B_{R}}-\hat{\phi}-L,
$$

for all $\hat{\phi}$ in $C_{\gamma}^{0}(\mathcal{D})$ with $\|\hat{\phi}\|_{\gamma}<C$. On $B_{R}, \phi_{n} \rightarrow \phi$ uniformly and so

$$
\sup _{\mathcal{D}}-\phi_{n}-L \rightarrow \sup _{\mathcal{D}}-\phi-L .
$$

This result completes the proof of Theorem 10 .

Applying this results we have the main result of this section 


\section{Theorem 6}

$$
\bar{H}=-\inf _{\mu \in \mathcal{M}_{0} \cap \mathcal{M}_{1}} \int L d \mu .
$$

Proof. We have by Theorem 10

$$
-\inf _{\mu \in \mathcal{M}_{0} \cap \mathcal{M}_{1}} \int L d \mu \leq \sup _{x} H\left(D_{x} u^{\epsilon}, x\right) \leq \bar{H}+O(\epsilon)
$$

in which $u^{\epsilon}=u * \eta_{\epsilon}$, with $\eta_{\epsilon}$ a standard mollifier, that is a positive smooth function, supported in a ball of radius $\epsilon$, with $\int \eta_{\epsilon}=1$.

Take a viscosity solution $u$ of

$$
H\left(D_{x} u, x\right)=\bar{H}
$$

Let $x^{\epsilon}$ be an admissible trajectory for which

$$
u\left(x^{\epsilon}(0)\right)-u\left(x^{\epsilon}\left(T_{\epsilon}\right)\right)-\int_{0}^{T_{\epsilon}} L\left(x^{\epsilon}, \dot{x}^{\epsilon}\right) \geq \bar{H} T_{\epsilon}+O(\epsilon),
$$

with $T_{\epsilon}=\frac{1}{\epsilon}$. Dividing the previous identity by $T_{\epsilon}$ and letting $\epsilon \rightarrow 0$ we get

$$
-\int L d \mu \geq \bar{H}
$$

in which $\mu$ is the measure defined by

$$
\int \psi(x, v) d \mu=\lim _{\epsilon \rightarrow 0} \frac{1}{T_{\epsilon}} \int_{0}^{T_{\epsilon}} \psi\left(x^{\epsilon}, \dot{x}^{\epsilon}\right)
$$

by taking an appropriate subsequence. This measure is, by the remark in the beginning of section 3, a vakonomic measure.

\section{Support of generalized Mather measures}

As was proved in the previous section, the generalized Mather measure is supported on a graph. The next proposition we develop some formal calculations that relate the support of the generalized Mather measure with the solution of Hamilton-Jacobi equations. Then in Theorem 7 we prove a rigorous version of this proposition.

Proposition 6 Let $\mu$ be a generalized Mather measure and $u$ a (smooth) viscosity solution of

$$
H\left(D_{x} u, x\right)=\bar{H} .
$$

Then $\mu$ is supported on the set $(x, v)$ in which $v$ is a minimizer of $D_{x} u f v+L(x, f v)$. 
Proof. If the proposition were false then there would be a set of positive $\mu$ measure for which

$$
H\left(D_{x} u, x\right)<-D_{x} u f v-L(x, f v) .
$$

Thus

$$
\bar{H}=\int H\left(D_{x} u, x\right) d \mu<-\int D_{x} u f v d \mu-\int L d \mu .
$$

But $\int D_{x} u f v d \mu=0$, and $-\int L d \mu=\bar{H}$, which yields a contradiction. Note that $L(x, f v)$ is strictly convex in $v$, therefore for each $x$ there exists a unique minimizer $v(x)$.

Before proving a rigorous version of the previous proposition we discuss some properties of $\bar{H}$ and its Legendre transform $\bar{L}$.

Proposition $7 \bar{H}(P)$ is convex in $P$ and superlinear.

Proof. Since

$$
\bar{H}(P)=\sup _{\mu \in \mathcal{M}_{0} \cap \mathcal{M}_{1}}-\int L+P v d \mu
$$

$\bar{H}(P)$ is the supremum of a family of convex functions - thus it is convex.

To show that $\bar{H}(P)$ is superlinear it suffices to observe that

$$
H\left(P+D_{x} u, x\right)=\frac{|f P|^{2}}{2}+f \operatorname{Pf} D_{x} u+\frac{\left|f D_{x} u\right|^{2}}{2}+V(x) \geq \gamma|P|^{2}+C
$$

in a set of positive measure, since $f$ is bracketing generating, and is non-negative elsewhere.

Let $\bar{L}(Q)$ denote the Legendre transform of $\bar{H}(P)$ :

$$
\bar{L}(Q)=\sup _{P}-\bar{H}(P)-P Q,
$$

which is finite because $\bar{H}$ is superlinear.

Proposition 8 Suppose $\mu^{*}$ is a minimizing measure corresponding to

$$
\inf _{\mu \in \mathcal{M}_{0} \cap \mathcal{M}_{1}} \int L(x, f v)+\operatorname{Pf}(x) v d \mu .
$$

Define

$$
Q^{*}=\int f(x) v d \mu^{*}
$$

Then

$$
\bar{L}\left(Q^{*}\right)=\int L(x, f v) d \mu^{*}=-\bar{H}(P)-P Q^{*} .
$$


Proof. Define

$$
\tilde{L}(Q)=\inf _{\mu \in \mathcal{M}_{0} \cap \mathcal{M}_{1} \int f(x) v d \mu=Q} \int L(x, f v) d \mu .
$$

Then we can write

$$
\bar{H}(P)=\sup _{Q}-P Q-\tilde{L}(Q)
$$

that is $\bar{H}(P)$ is the Legendre transform of $\tilde{L}(Q)$. Thus $\tilde{L}(Q)=\bar{L}(Q)$. Also

$$
\bar{H}(P)=-\bar{L}\left(Q^{*}\right)-P Q^{*}
$$

and so it follows

$$
\bar{L}\left(Q^{*}\right)+P Q^{*}=-\bar{H}(P)=\int L+P f v d \mu^{*} .
$$

Let $D_{P}^{-} \bar{H}(P)$ denote the subdifferential of $\bar{H}(P)$ at the point $P$. Since $\bar{H}$ is convex $D_{P}^{-} \bar{H}(P)$ is always non-empty. Then a standard convex analysis argument yields:

\section{Corollary 2}

$$
Q(P)=\int v d \mu^{*} \in D_{P}^{-} \bar{H}(P)
$$

The next theorem is a generalization of Fathi's results [Fat97a, Fat97b, Fat98a, Fat98b] (see also [E99], and [EG99a]) that asserts that the support of the Mather measure is a subset of the graph of the derivative of a viscosity solution of the Hamilton-Jacobi equation. Here we use the techniques from [EG99a].

Theorem 7 Let $\mu$ be a generalized Mather measure. Then $v=f\left(P+D_{x} u\right)$ $\mu$-almost everywhere.

Proof. Write

$$
\tilde{H}(v, x)=\frac{1}{2}|v|^{2}+V(x)
$$

for $v \in \mathbb{R}^{m}$. Note that

$$
\tilde{H}(f p, x)=H(p, x),
$$

and $\tilde{H}$ is strictly convex in $v$. Thus for any $v$ and $w$

$$
\tilde{H}(w, x) \geq \tilde{H}(v, x)+D_{v} \tilde{H}(v, x)(v-w)+\frac{\gamma}{2}|v-w|^{2} .
$$


Let $u$ be a viscosity solution. Let

$$
w=w(y)=f(y)\left(P+D_{x} u(y)\right),
$$

which, by Corollary 1 , is defined for almost every $y$, and let

$$
v=v(x)=\left[\eta_{\epsilon} *\left(f(\cdot)\left(P+D_{x} u(\cdot)\right)\right](x) .\right.
$$

Note that

$$
\int_{\mathbb{R}^{n}} D_{v} \tilde{H}(v(x), x)(v(x)-w(y)) \eta_{\epsilon}(x-y) d y=0
$$

and so

$$
\tilde{H}(v(x), x)+\beta_{\epsilon}(x) \leq \int_{\mathbb{R}^{n}} \eta_{\epsilon}(x-y) \tilde{H}(w(y), x) d y,
$$

with

$$
\beta_{\epsilon}(x)=\frac{\gamma}{2} \int_{\mathbb{R}^{n}} \eta_{\epsilon}(x-y)|v(x)-w(y)|^{2} d y .
$$

Observe that for almost every $y$

$$
\tilde{H}(w(y), x) \eta_{\epsilon}(x-y)=[\bar{H}(P)+O(\epsilon)] \eta_{\epsilon}(x-y),
$$

since for almost every $y$

$$
\tilde{H}(w(y), y)=\bar{H}(P),
$$

and $|x-y| \leq \epsilon$ whenever $\eta_{\epsilon}(x-y)$ is non-zero. So we have

$$
\tilde{H}(v(x), x)+\beta_{\epsilon}(x) \leq \bar{H}(P)+O(\epsilon),
$$

pointwise.

Also we have

$$
\begin{aligned}
& \frac{\gamma}{2} \int_{\mathcal{D}}|v(x)-z|^{2} d \mu(x, z) \leq \\
& \quad \leq \int_{\mathcal{D}} \tilde{H}(v(x), x)-\tilde{H}(z, x)-D_{v} \tilde{H}(z, x)(v(x)-z) d \mu(x, z),
\end{aligned}
$$

by strict convexity of $\tilde{H}$. Thus

$$
\begin{aligned}
& \frac{\gamma}{2} \int_{\mathcal{D}}|v(x)-z|^{2} d \mu(x, z)+\int_{\mathcal{D}} \beta_{\epsilon} d \mu(x, z) \leq \\
& \quad \leq \bar{H}(P)+\int_{\mathcal{D}}-\tilde{H}(z, x)-D_{v} \tilde{H}(z, x)(v(x)-z) d \mu(x, z)+O(\epsilon)
\end{aligned}
$$

Note that

$$
\begin{aligned}
& \int_{\mathcal{D}} D_{v} \tilde{H}(z, x) \eta_{\epsilon} *\left(f D_{x} u\right)(x) d \mu(x, z)= \\
& \quad=\int_{\mathcal{D}} D_{v} \tilde{H}(z, x) f(x) D_{x}\left(\eta_{\epsilon} * u\right)(x) d \mu(x, z)+O(\epsilon)=O(\epsilon)
\end{aligned}
$$


since

$$
\int_{\mathcal{D}} D_{v} \tilde{H}(z, x) f D_{x} \phi d \mu(x, z)=0
$$

for all periodic and smooth $\phi(x)$. Also

$$
\int_{\mathcal{D}} D_{v} \tilde{H}(z, x)\left(\eta_{\epsilon} * f P\right) d \mu(x, z)=Q P+O(\epsilon) .
$$

Note that

$$
-\tilde{H}(z, x)-D_{v} \tilde{H}(z, x) z=L(f z, x)
$$

which implies

$$
\int_{\mathcal{D}}-\tilde{H}(z, x)-D_{v} \tilde{H}(z, x) z d \mu(x, z)=\bar{L}(Q)
$$

Therefore

$$
\frac{\gamma}{2} \int_{\mathcal{D}}|v-z|^{2} d \mu+\int_{\mathcal{D}} \beta_{\epsilon} d \mu \leq \bar{H}(P)+\bar{L}(Q)+P Q+O(\epsilon)=O(\epsilon) .
$$

From this we conclude that $\beta_{\epsilon} \rightarrow 0$, through some subsequence, $\mu$-almost everywhere. So $\mu$-almost every point is a point of approximate continuity of $f D_{x} u$, thus

$$
\eta_{\epsilon} *\left(f D_{x} u\right) \rightarrow f D_{x} u,
$$

$\mu$-almost everywhere. From this we conclude $v=f D_{x} u \mu$-almost everywhere.

Corollary 3 For any $\phi(x)$ smooth and periodic we have

$$
\int f\left(f D_{x} u\right) D_{x} \phi d \mu=0 .
$$

\section{Applications}

In this section we discuss some applications of the generalized Mather measures. The firstly we prove asymptotic properties of certain vakonomic trajectories, then we study regularity for Hamilton-Jacobi equations and, finally we apply some of our results to the homogenization of Hamilton-Jacobi equations.

\subsection{Asymptotics}

The first applications is to prove that minimizing vakonomic trajectories have their asymptotic behavior controlled by the derivative of the effective Hamiltonian $\bar{H}$. 
Proposition 9 Suppose $\gamma(\cdot)$ is an optimal trajectory for the Hamilton-Jacobi equation, i.e.,

$$
u(x, P)=\int_{0}^{T} L(\gamma, \dot{\gamma})+P \dot{\gamma}+\bar{H}(P) d s+u(\gamma(T), P)
$$

Then

$$
\lim _{T \rightarrow \infty} \frac{\gamma(T)-\gamma(0)}{T}=-D_{P} \bar{H}(P),
$$

provided such a derivative exists.

Proof. For any other $P^{\prime}$

$$
u\left(x, P^{\prime}\right) \leq \int_{0}^{T} L(\gamma, \dot{\gamma})+P^{\prime} \dot{\gamma}+\bar{H}\left(P^{\prime}\right) d s+u\left(\gamma(T), P^{\prime}\right)
$$

Thus

$$
\left(P-P^{\prime}\right) \frac{\gamma(T)-\gamma(0)}{T}+\bar{H}(P)-\bar{H}\left(P^{\prime}\right) \leq O\left(\frac{1}{T}\right) .
$$

Let $P^{\prime} \rightarrow P$ and then $T \rightarrow \infty$.

\subsection{Regularity}

The last result on this paper uses a bootstrapping technique to show that whenever the viscosity solution $u$ is Lipschitz then the difference quotients of $f D_{x} u$ are $L^{2}$-Hölder continuous on the support of the Mather set.

Theorem 8 Let $\mu$ be a Mather measure and $u$ a Lipschitz periodic viscosity solution to

$$
H\left(D_{x} u, x\right)=\bar{H} .
$$

Then there exists a constant $C$ depending only on $H$ and on the Lipschitz constant of $u$ such that for all $w \in \mathbb{R}^{n}$

$$
\lim _{\epsilon \rightarrow 0} \int_{\mathbb{T}^{n}}\left|f(x+h w) D_{x} \hat{u}^{\epsilon}-f(x) D_{x} u(x)\right|^{2} d \mu \leq C|h|,
$$

in which $\hat{u}=u(x+h w)$ and $\hat{u}^{\epsilon}=\eta_{\epsilon} * \hat{u}$.

Proof. Fix $w \in \mathbb{R}^{m}$ and define $\hat{u}(x)=u(x+h w)$. Then, almost everywhere,

$$
H\left(D_{x} \hat{u}, x+h w\right)=\bar{H},
$$

and by setting $\hat{u}^{\epsilon}=\eta_{\epsilon} * \hat{u}$, we have

$$
H\left(D_{x} \hat{u}^{\epsilon}, x+h w\right) \leq \bar{H}+C \epsilon .
$$


Therefore

$$
\begin{aligned}
\frac{1}{2} \mid f(x+h w) D_{x} \hat{u}^{\epsilon}- & \left.f(x) D_{x} u\right|^{2}+ \\
& \left.+f(x) D_{x} u\left[f(x+h w) D_{x} \hat{u}^{\epsilon}-f(x) D_{x} u\right)\right] \leq \\
& \leq H\left(D_{x} \hat{u}^{\epsilon}, x+h w\right)-H\left(D_{x} u, x\right)+C h \leq \\
& \leq C(\epsilon+h)
\end{aligned}
$$

Integrating with respect to $\mu$ we obtain

$$
\begin{aligned}
\frac{\gamma}{2} \int_{\mathbb{T}^{n}} \mid & f(x+h w) D_{x} \hat{u}^{\epsilon}-\left.f(x) D_{x} u^{\epsilon}\right|^{2} d \mu \leq \\
& \leq-h \int_{\mathbb{T}^{n}} f(x) D_{x} u(f(x+h w)-f(x)) D_{x} \hat{u}^{\epsilon} d \mu+C(\epsilon+h)= \\
& =C(\epsilon+h) .
\end{aligned}
$$

The result follows from sending $\epsilon \rightarrow 0$.

Finally we we would like to point out that this result may not be optimal since formal computations seem to indicate that on the Mather set we should have

$$
\int\left|f D_{x}\left(f D_{x} u\right)\right|^{2} d \mu \leq C .
$$

However, we were unable to improve the previous proof to obtain a result like the previous one.

Proposition 10 Suppose $u$ is a smooth solution of

$$
H\left(D_{x} u, x\right)=\bar{H} .
$$

Let $\mu$ be a corresponding Mather measure. Then

$$
\int\left|f D_{x}\left(f D_{x} u\right)\right|^{2} d \mu \leq C .
$$

Proof. Apply $f D_{x}$ twice to the equation

$$
\frac{\left|f D_{x} u\right|^{2}}{2}+V(x)=\bar{H}
$$

to obtain

$$
\left|f D_{x}\left(f D_{x} u\right)\right|^{2}+f D_{x} u f D_{x}\left(f D_{x}\left(f D_{x} u\right)\right)+\left(f D_{x}\right)^{2} V=0 .
$$

Since $\int f D_{x} u f D_{x} \phi d \mu=0$ for all $\phi$ periodic, we have

$$
\int f D_{x} u f D_{x}\left(f D_{x}\left(f D_{x} u\right)\right) d \mu=0,
$$

and since $V$ is smooth the result follows. 


\section{References}

[AKN97] V. I. ARNOLD, V. V. KOZLOV and A. I. NEISHTADT, Mathematical aspects of classical and celestial mechanics. Springer-Verlag, Berlin, 1997. Translated from the 1985 Russian original by A. Iacob, Reprint of the original English edition from the series Encyclopaedia of Mathematical Sciences [Dynamical systems. III, Encyclopaedia Math. Sci., 3, Springer, Berlin, 1993; MR 95d:58043a].

[Bac86] A. BACCIOTTI, Fondamenti geometrici della teoria della controllabilità. Quaderni U.M.I. Pitagora, no. 31, Bologna, 1986.

[BCD97] M. BARDI and I. CAPUZZO-DOLCETTA, Optimal control and viscosity solutions of Hamilton-Jacobi-Bellman equations. Birkhäuser Boston Inc., Boston, MA, 1997. With appendices by Maurizio Falcone and Pierpaolo Soravia.

[Car09] C. CARATHÉODORY, Untersuchungen über die grundlagen der thermodynamik. MATH. ANN., 67 (1909), 355-386.

[Cho39] W. L. CHOW, Uber systeme von lineaaren partiellen differentialgleichungen ester orduung. Math. Ann., 117 (1938), 98-105.

[CIPP98] G. CONTRERAS, R. ITURRIAGA, G. P. PATERNAIN and M. PATERNAIN, Lagrangian graphs, minimizing measures and Mañé's critical values. Geom. Funct. Anal., 8(5) (1998), 788-809.

[E99] E. WEINAN, Aubry-Mather theory and periodic solutions of the forced Burgers equation. Comm. Pure Appl. Math., 52(7) (1999), 811-828.

[EG99a] L. C. EVANS and D. GOMES, Effective Hamiltonians and averaging for Hamiltonian dynamics I. Preprint, 1999.

[EG99b] L. C. EVANS and D. GOMES, Effective Hamiltonians and averaging for Hamiltonian dynamics II. Preprint, 1999.

[EJ89] L. C. EVANS and M. R. JAMES, The Hamilton-Jacobi-Bellman equation for time-optimal control. SIAM J. Control Optim., 27(6) (1989), $1477-1489$.

[Fat97a] ALBERT FATHI, Solutions KAM faibles conjuguées et barrières de Peierls. C. R. Acad. Sci. Paris Sér. I Math., 325(6) (1997), 649-652.

[Fat97b] ALBERT FATHI, Théorème KAM faible et théorie de Mather sur les systèmes lagrangiens. C. R. Acad. Sci. Paris Sér. I Math., 324(9) (1997), 1043-1046. 
[Fat98a] ALBERT FATHI, Orbite hétéroclines et ensemble de Peierls. $C . R$. Acad. Sci. Paris Sér. I Math., 326 (1998), 1213-1216.

[Fat98b] ALBERT FATHI, Sur la convergence du semi-groupe de Lax-Oleinik. C. R. Acad. Sci. Paris Sér. I Math., 327 (1998), 267-270.

[Fle89] WENDELL H. FLEMING, Generalized solutions and convex duality in optimal control. In Partial differential equations and the calculus of variations, Vol. I, pages 461-471. Birkhäuser Boston, Boston, MA, 1989.

[FS93] WENDELL H. FLEMING and H. METE SONER, Controlled Markov processes and viscosity solutions. Springer-Verlag, New York, 1993.

[FS04] ALBERT FATHI and ANTONIO SICONOLFI, Existence of $C^{1}$ critical subsolutions of the Hamilton-Jacobi equation. Invent. Math., 155(2) (2004), 363-388.

[FS05] ALBERT FATHI and ANTONIO SICONOLFI, PDE aspects of AubryMather theory for quasiconvex Hamiltonians. Calc. Var. Partial Differential Equations, 22(2) (2005), 185-228.

[FV88] WENDELL H. FLEMING and DOMOKOS VERMES, Generalized solutions in the optimal control of diffusions. In Stochastic differential systems, stochastic control theory and applications (Minneapolis, Minn., 1986), pages 119-127. Springer, New York, 1988.

[FV89] WENDELL H. FLEMING and DOMOKOS VERMES, Convex duality approach to the optimal control of diffusions. SIAM J. Control Optim., 27(5) (1989), 1136-1155.

[Gom00] D. GOMES, Viscosity solutions of Hamilton-Jacobi equations, and asymptotics for Hamiltonian systems. Preprint, 2000.

[Gom02] D. GOMES, A stochastic analog of Aubry-Mather theory. To appear in Nonlinearity, 2002.

[Gyu84] É. GYURKOVICS, Hölder condition for the minimum time function of linear systems. In System modelling and optimization (Copenhagen, 1983), pages 383-392. Springer, Berlin, 1984.

[Gyu87] É. GYURKOVICS, Hölder condition for the minimum time function. Problems Control Inform. Theory/Problemy Upravlen. Teor. Inform., 16(1) (1987), 61-72.

[HH70] G. W. HAYNES and H. HERMES, Nonlinear controllability via Lie theory. SIAM J. Control, 8 (1970), 450-460. 
[JKM99] H. R. JAUSLIN, H. O. KREISS and J. MOSER, On the forced Burgers equation with periodic boundary conditions. In Differential equations: La Pietra 1996 (Florence), pages 133-153. Amer. Math. Soc., Providence, RI, 1999.

[JLS88] R. JENSEN, P.-L. LIONS and P. E. SOUGANIDIS, A uniqueness result for viscosity solutions of second order fully nonlinear partial differential equations. Proc. Amer. Math. Soc., 102(4) (1988), 975-978.

[KO01] IVAN KUPKA and WALDYR M. OLIVA, The non-holonomic mechanics. J. Differential Equations, 169(1) (2001), 169-189. Special issue in celebration of Jack K. Hale's 70th birthday, Part 3 (Atlanta, GA/Lisbon, 1998).

[Liv80] A. A. LIVEROVSKII , Some properties of the Bellman function for linear and symmetric polysystems. Differentsial' nye Uravneniya, 16(3) (1980), 414-423, 571-572.

[LS95] WENSHENG LIU and HÉCTOR J. SUSSMAN, Shortest paths for sub-Riemannian metrics on rank-two distributions. Mem. Amer. Math. Soc., 118(564) (1995), $\mathrm{x}+104$.

[LV80] R. M. LEWIS and R. B. VINTER, Relaxation of optimal control problems to equivalent convex programs. J. Math. Anal. Appl., 74(2) (1980), 475-493.

[Mn96] RICARDO MAÑÉ, Lagrangian flows: the dynamics of globally minimizing orbits. In International Conference on Dynamical Systems (Montevideo, 1995), pages 120-131. Longman, Harlow, 1996.

[Ran82] MICHAËL RANGUIN, Propriété holdérienne de la fonction temps minimal d'un système linéaire autonome. RAIRO Automat., 16(4) (1982), 329-340.

[Roc66] R. T. ROCKAFELLAR, Extension of Fenchel's duality theorem for convex functions. Duke Math. J., 33 (1966), 81-89.

[Sor92] P. SORAVIA, Hölder continuity of the minimum-time function for $C^{1}$ manifold targets. J. Optim. Theory Appl., 75(2) (1992), 401-421.

[Str86] ROBERT S. STRICHARTZ, Sub-Riemannian geometry. J. Differential Geom., 24(2) (1986), 221-263.

[Str89] ROBERT S. STRICHARTZ, Corrections to: "Sub-Riemannian geometry" [J. Differential Geom. 24(2) (1986), 221-263; MR 88b:53055]. J. Differential Geom., 30(2) (1989), 595-596. 
[VL78a] RICHARD B. VINTER and RICHARD M. LEWIS, The equivalence of strong and weak formulations for certain problems in optimal control. SIAM J. Control Optim., 16(4) (1978), 546-570.

[VL78b] RICHARD B. VINTER and RICHARD M. LEWIS, A necessary and sufficient condition for optimality of dynamic programming type, making no a priori assumptions on the controls. SIAM J. Control Optim., 16(4) (1978), 571-583.

Received 16 March 2005; accepted 16 June 2005

To access this journal online: http://www.birkhauser.ch 